\title{
Association of FTO methylation level with incident type 2 diabetes mellitus: a nested case-control study within the Rural Chinese Cohort Study
}

Shengbing Huang

Zhengzhou University

Qing Chen

Department of Mental Health, Bao an Chronic Diseases Prevent and Cure Hospital

Dongdong Zhang

Zhengzhou University

Cheng Cheng

Zhengzhou University

Chunmei Guo

Zhengzhou University

Quanman Li

Zhengzhou University

Qionggui Zhou

Shenzhen University

Gang Tian

Zhengzhou University

Ranran Qie

Zhengzhou University

Minghui Han

Zhengzhou University

Xiaoyan Wu

Shenzhen University

Yang Zhao

Zhengzhou University

Xingjin Yang

Zhengzhou University

Yifei Feng

Zhengzhou University

\section{Yang Li}

Shenzhen University

Pei Qin 
Shenzhen University

\section{Yang Zhao}

Zhengzhou University

YanYan Zhang

Shenzhen University

\section{Yuying Wu}

Shenzhen University

Dechen Liu

Zhengzhou University

Ming Zhang

Shenzhen University

Dongsheng Hu ( $\square$ dongshenghu563@126.com )

Zhengzhou University

Jie Lu

Zhengzhou University

\section{Research}

Keywords: FTO, Methylation level, SNP, T2DM, Haplotype

Posted Date: February 12th, 2020

DOI: https://doi.org/10.21203/rs.2.23334/v1

License: (1) (1) This work is licensed under a Creative Commons Attribution 4.0 International License.

Read Full License 


\section{Abstract}

Objectives

This study aimed to investigate the association of FTO methylation level with type 2 diabetes mellitus (T2DM).

Methods

We conducted a nested case - control study for DNA methylation of FTO in Chinese pople identified from the Rural Chinese Cohort Study with a 6-year follow-up. Controls were matched to the cases on a 1:1 basis by age, sex, ethnicity, marital status, and residence. Unconditional multivariate logistic regression models were used to calculate odds ratios (ORs) and 95\% confidence intervals (Cls) for association with Tag-single nucleotide polymorphisms (SNPs) and cytosine guanine (CpG) locus. Haploview was used to analyze the association of possible haplotypes with T2DM. Generalized Multifactor Dimensionality Reduction was used to explore the potential interaction.

Results

We found a significant association for the CpG9 locus located in the promoter region of FTO with T2DM. With CpG9, the risk of T2DM was $1.84(1.21-2.80)$ after adjusting for potential confounders. The TagSNPs (rs72803657, rs1558902, rs17817449, rs11076023) nor were possible haplotypes associated with T2DM in the strong linkage disequilibrium region. We found no significant interaction between obesity indexes, serum lipid levels and methylation levels.

Conclusions

Our study identified a CpG locus located in the promoter region of FTO that altered the T2DM risk independent of body mass index, but the variants of FTO were not significantly associated with T2DM. Our study might provide new insights into the pathways of FTO with T2DM and offer new opportunities for risk stratification and prevention of T2DM among rural Chinese population.

\section{Introduction}

The world's population with type 2 diabetes mellitus (T2DM) was predicted to increase to 642 million by 2040, especially in low and middle-income countries such as China [1], because of urbanization [2] and transition to a western lifestyle [3]. According to the IDF diabetes atlas 2017, more than 114.4 million individuals experienced T2DM in 2017 and the number would reach 129.7 million in 2045 in China [2]. T2DM has reached epidemic proportions among general adults in China, which has led to severe social, medical, and economical burdens. Thus, it is important to explore the causes and intervention of T2DM.

The fat mass and obesity-associated gene (FTO) is the first gene shown to contribute to non-syndromic human obesity $[4,5]$. It was widely verified with variety of obesity traits throughout the life courses and 
across diverse ancestries[6]. However, the association with T2DM remains controversial [7-9]. A prospective population-based cohort study recruiting 25,631 participants in Norwich suggested FTO variants associated with T2DM [10], whereas a case-control study including 3,040 participants in Taiwan suggested no association between FTO variants and T2DM [11]. Furthermore, few studies included the epigenetic context of FTO in the process of T2DM. DNA methylation might provide new insights into the pathways underlying T2DM [12]. A case-control study found a $\mathrm{CpG}$ site in the first intron of FTO showed small (3.35\%) but significant $(P=0.000021)$ hypomethylation of cases relative to controls [13], and a cross-sectional study also suggested that a methylation difference between diabetic patients and controls for one CpG locus located in FTO was apparent in young individuals [14], another case-control study suggested that the association with T2DM tended toward significance between T2DM patients and controls [15]. The conclusions of these studies are controversial and as far as we know, none of the studies directly analyzed the effect of FTO methylation level on T2DM incident in the rural Chinese population.

Therefore, the aim of our study was to evaluate the associations of FTO methylation level with T2DM based on a nested case-control study in a rural Chinese population.

\section{Reseach Design And Methods Study population}

In this nested case-control study, we explored an epigenome-wide association of DNA methylation in people with incident T2DM who were identified from the Rural Chinese Cohort Study with 6 follow-up years. Details about the design, participants, methods, and measurements of the cohort study have been previously described [16]. Participants with incident T2DM were defined as those who did not have T2DM at baseline, but T2DM developed during follow-up. Controls were participants who did not have T2DM at both baseline and follow-up. After excluding participants with baseline T2DM $(n=1,499)$, T1DM $(n=13)$, cancer $(n=28)$, stroke $(n=372)$, myocardial infarction $(n=183)$, chronic obstructive pulmonary diseases $(n=353)$, and chronic kidney disease $(n=294), 14,523$ non-diabetic participants at baseline were the target participants for the current analysis. During the follow-up, 707 new cases of T2DM were ascertained. Among the new-onset 707 diabetic cases and 13,816 non-diabetic participants, 293 pairs of study population were selected using the case-control matching method for DNA methylation and SNPs measurement [17]. Controls were matched to cases on a 1:1 basis by age (birth at the same year), sex, ethnicity, marital status, and residence (live in the same village). Finally, 293 pairs of cases and controls with complete information and blood samples were included in this nested case-control study (Figure S1).

\section{Data Collection And Relevant Definitions And Diagnosis Criteria}


We used a standard questionnaire to collect information on demographics (age, sex, ethnicity, residence and marital status), lifestyle behaviors (smoking, alcohol drinking, and physical activity), and personal and family medical history at baseline. Smoking was defined as ever smoking at least 100 cigarettes during the lifetime [18]. Alcohol drinking was defined as consuming drinking at least 12 times during the last year [18]. Physical activity level was classified as low, moderate and high according to the International Physical Activity Questionnaire [19]. The detailed information on body weight, height, waist circumference (WC), body mass index (BMI), systolic blood pressure (SBP), diastolic blood pressure (DBP), fasting plasma glucose (FPG), total cholesterol (TC), triglycerides (TG), and high-density lipoprotein cholesterol (HDL-C) were described as previously published [20]. BMI was calculated as weight $(\mathrm{kg}) /$ height $(\mathrm{m})^{2}$. Low-density lipoprotein cholesterol (LDL-C) was calculated by the Fried Ewald formula [21].

T2DM was defined as fasting glucose $\geq 7.0 \mathrm{mmol} / \mathrm{L}$ and/or the use of insulin or oral hypoglycemic agents, and/or a self-reported history of diabetes, which agreed with the diagnostic criteria of T2DM at both baseline and follow-up examination [22].

\section{Quantitative DNA Methylation Measurement}

DNA samples were extracted from baseline fasting peripheral-blood leucocytes by the automated nucleic acid extraction system (BioTeke Corp., Beijing). The target sequence covered 428 bp (Chr16:5370350953703936) located in the promoter region of FTO. The sequenom ${ }^{\circledR}$ EpiDesigner system was used to design the primer sequences: forward 5'-aggaagagagTTTGTAGGATTTGGATAGAGATGGT-3' and reverse 3'-cagtaatacgactcactatagggagaagg ctAAATCCAAAAAAAACTACATTTCCC-5'. After genomic DNA was treated with bisulfite, PCR was used to amplify the target sequence, followed by removing 5 '- and 3'phosphate groups from the products by using shrimp alkaline phosphate. EpiTYPER biochemistry began with bisulfite treatment of genomic DNA, followed by PCR amplification of target regions, then DNA transcription in vitro, and Uracil-specific DNA cleavage. Finally, the mass spectra of cleavage products were analyzed by using MALDI-TOF mass spectrometry based on the MassARRAY System (Bio Miao Biological Technology, Beijing), and the level of DNA methylation was measured by MassARRAY EpiTYPER analysis (Agena Bioscience, San Diego, CA). DNA methylation was detected in case-control pairs.

\section{Tag-SNP Selection And Genotyping}

The Tag-SNPs were selected from an extensive review of the literature and from the HapMap and NCBI databases. The selection criterion was minor allele frequency (MAF) $>0.01$. Eleven SNPs in FTO (rs72803657, rs9939609, rs1121980, rs17817449, rs8050136, rs9940128, rs9926289, rs11076023, rs1558902, rs1421085, and rs9941349) were selected for our study. All SNPs met Hardy-Weinberg equilibrium (HWE). Finally, four Tag-SNPs were selected in our study based on the linkage disequilibrium $(L D)$ analysis $\left(r^{2} \geq 0.8\right)$. Specific primers were designed by using Assay design3.1 software (Sequenom 
Inc). Genotyping and PCR involved using a MassARRAY Genotyping system (Agena Inc). We re-genotyped approximately $4 \%$ of random samples to control the quality. The agreement of the genotypes determined for the blind control samples was $100 \%$.

\section{Statistical analysis}

Baseline characteristics were compared between T2DM cases and controls. Continuous variables are shown as median (interquartile range) for data with skewed distribution, and the Wilcoxon Rank Sum Test was used to assess differences in these data. Continuous variables with normal distribution are shown as mean (SD) and were analyzed by t-test. Categorical variables are shown as number (\%) and were analyzed by chi-square test. Chi-square test was used to test for HWE among controls. The association between SNPs and T2DM was assessed by multiple logistic regression models with a dominant genetic model of Tag-SNPs. Haploview was used for analysis of LD and haplotype analyses was used to explore the association of possible haplotypes with T2DM. The methylation level was non-normally distributed and was compared by Wilcoxon Rank Sum Test. Considering important nonmatching variables between cases and controls apart from age, sex, marital status and residence region[8], we used unconditional logistic regression models to evaluate the association between methylation level and risk of T2DM. We adopted three models: 1) unadjusted; 2) adjusted for smoking, alcohol drinking, physical activity, SBP, FPG; and 3) adjusted for smoking, drinking, physical activity, SBP, BMI, and FPG, TG and HDL-C levels. Spearman correlation analysis was used to explore the association with T2DM-related quantitative phenotypes. Wilcoxon-Rank Test was used to compare the methylation level in different SNP alleles. Finally, we used Generalized Multifactor Dimensionality Reduction (GMDR) to explore potential interactions of Tag-SNPs, anthropometry indexes (BMI, WC, and TC, TG, HDL-C and LDL-C levels), environmental factors (smoking, alcohol drinking, physical activity) with CpG locus.

\section{Results}

Baseline demographic characteristics of the 293 incident T2DM cases and 293 controls were shown in Table 1 . The mean age was $52.29 \pm 9.50$ years and the proportion of females was $65.53 \%$. Physical activity, smoking, alcohol drinking, and levels of TC and LDL-C did not differ between cases and controls; family history of diabetes, BMI, SBP, DBP, and FPG, TG levels were significantly higher and HDL-C level was lower for cases than controls. 
Table 1

Baseline demographic characteristics of study participants.

\begin{tabular}{|c|c|c|c|}
\hline Characteristics & Controls $(n=293)$ & Cases $(n=293)$ & $P$ value \\
\hline Women $(n, \%)$ & $192(65.53)$ & $192(65.53)$ & - \\
\hline Age (years) & $52.29 \pm 9.50$ & $52.29 \pm 9.50$ & - \\
\hline Smoking (n, \%) & $77(26.28)$ & $66(22.53)$ & $0.21^{\mathrm{a}}$ \\
\hline Alcohol drinking (n, \%) & $25(8.53)$ & $28(9.56)$ & $0.67^{\mathrm{a}}$ \\
\hline Physical activity (n, \%) & & & $0.94^{\mathrm{a}}$ \\
\hline Low & $82(27.99)$ & $85(29.01)$ & \\
\hline Moderate & $55(18.77)$ & $56(19.11)$ & \\
\hline High & $156(53.24)$ & $152(51.88)$ & \\
\hline Family history of diabetes (n, \%) & $34(13.23)$ & $147(54.85)$ & $<0.01^{\mathrm{a}}$ \\
\hline $\mathrm{BMI}\left(\mathrm{kg} / \mathrm{m}^{2}\right)$ & $22.08(24.59-26.82)$ & $23.51(25.78-28.50)$ & $<0.01^{\mathrm{b}}$ \\
\hline WC $(\mathrm{cm})$ & $83.71 \pm 10.14$ & $88.22 \pm 11.07$ & $<0.01^{\mathrm{c}}$ \\
\hline $\mathrm{SBP}(\mathrm{mm} \mathrm{Hg})$ & $123.00(112.33-138.67)$ & $129.67(116.67-143.67)$ & $<0.01^{\mathrm{b}}$ \\
\hline $\mathrm{DBP}(\mathrm{mm} \mathrm{Hg})$ & $79.64(71.33-86.33)$ & $82.82(74.67-90.33)$ & $<0.01^{\mathrm{b}}$ \\
\hline $\mathrm{TC}(\mathrm{mmol} / \mathrm{L})$ & $4.46(3.99-5.04)$ & $4.62(4.05-5.16)$ & $0.09^{b}$ \\
\hline $\mathrm{TG}(\mathrm{mmol} / \mathrm{L})$ & $1.38(0.99-1.94)$ & $1.73(1.15-2.36)$ & $<0.01^{\mathrm{b}}$ \\
\hline $\mathrm{HDL}-\mathrm{C}(\mathrm{mmol} / \mathrm{L})$ & $1.16(1.00-1.34)$ & $1.09(0.95-1.26)$ & $<0.01^{\mathrm{b}}$ \\
\hline LDL-C (mmol/L) & $2.20(2.60-3.00)$ & $2.20(2.60-3.10)$ & $0.52^{\mathrm{b}}$ \\
\hline
\end{tabular}

Data are expressed as mean \pm SD, median (inter-quartile range), or $n(\%)$ as appropriate.

Abbreviations:

BMI, body mass index; WC, waist circumstance; SBP, systolic blood pressure; DBP, diastolic blood pressure; TC, total cholesterol; TG, triglycerides; HDL-C, high density lipoprotein cholesterol; LDL-C, low density lipoprotein cholesterol; FPG, fasting plasma glucose.

a. Chi-square test.

b: Wilcoxon Rank Sum Test.

c. T-test. 


\begin{tabular}{|c|c|c|c|}
\hline Characteristics & Controls $(n=293)$ & Cases $(n=293)$ & $P$ value \\
\hline FPG (mmol/L) & $5.33(5.05-5.70)$ & $5.95(5.46-6.42)$ & $<0.01^{\mathrm{b}}$ \\
\hline \multicolumn{4}{|c|}{ Data are expressed as mean $\pm S D$, median (inter-quartile range), or $n(\%)$ as appropriate. } \\
\hline \multicolumn{4}{|l|}{ Abbreviations: } \\
\hline \multicolumn{4}{|c|}{$\begin{array}{l}\text { BMI, body mass index; WC, waist circumstance; SBP, systolic blood pressure; DBP, diastolic blood } \\
\text { pressure; TC, total cholesterol; TG, triglycerides; } \mathrm{HDL}-\mathrm{C} \text {, high density lipoprotein cholesterol; LDL-C, low } \\
\text { density lipoprotein cholesterol; FPG, fasting plasma glucose. }\end{array}$} \\
\hline \multicolumn{4}{|c|}{ a: Chi-square test. } \\
\hline \multicolumn{4}{|c|}{ b. Wilcoxon Rank Sum Test. } \\
\hline c: T-test. & & & \\
\hline
\end{tabular}

Cases and controls significantly differed in CpG9 methylation level (Fig. 1). As compared with methylation level < 75\%, CpG9 level $\geq 75 \%$ was associated with T2DM: OR 1.64 (95\% Cl, 1.13-2.38) (Table 2). After adjusting for smoking, drinking, physical activity, SBP, BMI, and FPG, TG and HDLC levels, the association remained robust $(\mathrm{OR}=1.84,95 \% \mathrm{Cl}, 1.21-2.80)$. To explore the possible mechanisms of the $\mathrm{CpG}$ locus on T2DM susceptibility, we tested the association of CpG9 with the T2DM-related quantitative phenotypes (BMI, WC, and levels of TC, LDL-C, HDL-C, and TG) by Spearman correlation coefficients in the combined sample (normal glucose tolerance [NGT] + T2DM). However, we found no association between methylation level and obesity associated indexes (Table S1) or between Tag-SNPs and methylation level of CpG9 (Table S2). 
Table 2

Association of methylation level with type 2 diabetes mellitus (T2DM).

\begin{tabular}{|c|c|c|c|c|c|}
\hline \multirow[t]{2}{*}{ Loci } & \multirow{2}{*}{$\begin{array}{l}\text { Case/ } \\
\text { control }\end{array}$} & \multirow{2}{*}{$\begin{array}{l}\text { Lower methylation level } \\
\text { (reference) }\end{array}$} & \multicolumn{3}{|l|}{ OR $(95 \% \mathrm{Cl})$} \\
\hline & & & Model 1 & Model 2 & Model 3 \\
\hline $\begin{array}{l}\text { CpG1 } \\
\text { Chr16:53703596 }\end{array}$ & 280 & $<2 \%$ & $\begin{array}{l}1.21(0.86- \\
1.70)\end{array}$ & $\begin{array}{l}1.18(0.83- \\
1.67)\end{array}$ & $\begin{array}{l}1.06(0.72- \\
1.56)\end{array}$ \\
\hline $\begin{array}{l}\text { CpG2 } \\
\text { Chr16:53703611 }\end{array}$ & 279 & $<22 \%$ & $\begin{array}{l}1.12(0.79- \\
1.59)\end{array}$ & $\begin{array}{l}1.06(0.74- \\
1.52)\end{array}$ & $\begin{array}{l}1.16(0.78- \\
1.72)\end{array}$ \\
\hline $\begin{array}{l}\text { CpG3 } \\
\text { Chr16:53703666 }\end{array}$ & 243 & $<3 \%$ & $\begin{array}{l}1.26(0.88- \\
1.81)\end{array}$ & $\begin{array}{l}1.36(0.93- \\
1.97)\end{array}$ & $\begin{array}{l}1.20(0.80- \\
1.79)\end{array}$ \\
\hline $\begin{array}{l}\text { CpG4 } \\
\text { Chr16:53703708 }\end{array}$ & 270 & $<5 \%$ & $\begin{array}{l}0.83(0.57- \\
1.21)\end{array}$ & $\begin{array}{l}0.84(0.57- \\
1.23)\end{array}$ & $\begin{array}{l}0.82(0.53- \\
1.25)\end{array}$ \\
\hline $\begin{array}{l}\text { CpG5 } \\
\text { Chr16:53703716 }\end{array}$ & 274 & $<9 \%$ & $\begin{array}{l}0.83(0.57- \\
1.19)\end{array}$ & $\begin{array}{l}0.84(0.58- \\
1.22)\end{array}$ & $\begin{array}{l}0.77(0.51- \\
1.17)\end{array}$ \\
\hline $\begin{array}{l}\text { CpG6_8* } \\
\text { Chr16:53703731 } \\
\text { /53703736 } \\
/ 53703738\end{array}$ & 280 & $<5 \%$ & $\begin{array}{l}1.20(0.85- \\
1.68)\end{array}$ & $\begin{array}{l}1.20(0.85- \\
1.70)\end{array}$ & $\begin{array}{l}1.05(0.72- \\
1.54)\end{array}$ \\
\hline $\begin{array}{l}\text { CpG9 } \\
\text { Chr16:53703794 }\end{array}$ & 280 & $<5 \%$ & $\begin{array}{l}1.64(1.13- \\
2.38)\end{array}$ & $\begin{array}{l}1.64(1.12- \\
2.40)\end{array}$ & $\begin{array}{l}1.84(1.21- \\
2.80)\end{array}$ \\
\hline $\begin{array}{l}\text { CpG10 } \\
\text { Chr16:53703805 }\end{array}$ & 266 & $<3 \%$ & $\begin{array}{l}1.42(1.00- \\
2.01)\end{array}$ & $\begin{array}{l}1.38(0.97- \\
1.98)\end{array}$ & $\begin{array}{l}1.33(0.90- \\
1.97)\end{array}$ \\
\hline $\begin{array}{l}\text { CpG11 } \\
\text { Chr16:53703832 }\end{array}$ & 280 & $<11.5 \%$ & $\begin{array}{l}1.02(0.70- \\
1.48)\end{array}$ & $\begin{array}{l}0.96(0.65- \\
1.41)\end{array}$ & $\begin{array}{l}1.10(0.72- \\
1.68)\end{array}$ \\
\hline $\begin{array}{l}\text { CpG12 } 13^{*} \\
\text { Chr16:53703843 } \\
\text { /53703846 }\end{array}$ & 278 & $<1 \%$ & $\begin{array}{l}1.02(0.70- \\
1.48)\end{array}$ & $\begin{array}{l}1.07(0.73- \\
1.57)\end{array}$ & $\begin{array}{l}1.01(0.67- \\
1.54)\end{array}$ \\
\hline $\begin{array}{l}\text { CpG14-17* } \\
\text { Chr16:53703852 } \\
\text { /53703857 } \\
/ 53703861 \\
/ 53703869\end{array}$ & 280 & $<4 \%$ & $\begin{array}{l}1.00(0.72- \\
1.39)\end{array}$ & $\begin{array}{l}1.00(0.71- \\
1.39)\end{array}$ & $\begin{array}{l}0.97(0.67- \\
1.40)\end{array}$ \\
\hline $\begin{array}{l}\text { CpG18 } \\
\text { Chr16:53703880 }\end{array}$ & 274 & $<9 \%$ & $\begin{array}{l}0.83(0.57- \\
1.19)\end{array}$ & $\begin{array}{l}0.84(0.58- \\
1.22)\end{array}$ & $\begin{array}{l}0.77(0.51- \\
1.17)\end{array}$ \\
\hline
\end{tabular}

OR: odds ratio, Cl: confidence interval;

Model 1: unadjusted;

Model 2: adjusted for smoking, alcohol drinking, physical activity, SBP, FPG;

Model 3: adjusted for smoking, alcohol drinking, physical activity, SBP, BMI, levels of FPG, TG and HDL-C. 


\begin{tabular}{|llllll}
\hline Loci & $\begin{array}{l}\text { Case/ } \\
\text { control }\end{array}$ & $\begin{array}{l}\text { Lower methylation level } \\
\text { (reference) }\end{array}$ & \multicolumn{2}{l}{ OR (95\% Cl) } & \\
\cline { 3 - 6 } & & & Model 1 & Model 2 & Model 3 \\
\hline CpG19 & 280 & $<3 \%$ & $1.07(0.74-$ & $1.06(0.73-$ & $0.96(0.63-$ \\
Chr16:53703902 & & & $1.56)$ & $1.55)$ & $1.47)$ \\
\hline
\end{tabular}

OR: odds ratio, Cl: confidence interval;

Model 1: unadjusted;

Model 2: adjusted for smoking, alcohol drinking, physical activity, SBP, FPG;

Model 3: adjusted for smoking, alcohol drinking, physical activity, SBP, BMI, levels of FPG, TG and HDL-C.

Allele frequencies of the Tag-SNPs and P value of HWE deviation are in Table 3. After adjusting for smoking, alcohol drinking, physical activity, SBP, FPG, and BMI, we still found no significant association between Tag-SNPs and T2DM. Figure S2 illustrates the linkage disequilibrium analysis of the 11 SNPs. Strong linkage disequilibrium was observed in block 1. Furthermore, we did not find any possible haplotype associated with T2DM (Table S3). 
Table 3

Association of Tag-SNPs with T2DM under dominant genetic models.

\begin{tabular}{|c|c|c|c|c|c|c|}
\hline \multirow[t]{2}{*}{ SNPs } & \multirow{2}{*}{$\begin{array}{l}\text { Case/ } \\
\text { control }\end{array}$} & \multirow[t]{2}{*}{ Genotype } & \multirow[t]{2}{*}{$\mathrm{P}^{\mathrm{a}}$} & \multicolumn{3}{|l|}{ OR $(95 \% \mathrm{Cl})$} \\
\hline & & & & Model 1 & Model 2 & Model 3 \\
\hline rs72803657 & 283 & GG/GT/TT & 0.56 & $\begin{array}{l}1.07(0.72- \\
1.59)\end{array}$ & $\begin{array}{l}1.05(0.70- \\
1.58)\end{array}$ & $\begin{array}{l}1.00(0.64- \\
1.56)\end{array}$ \\
\hline Case & & $9 / 56 / 218$ & & & & \\
\hline Control & & $5 / 57 / 221$ & & & & \\
\hline rs1558902 & 271 & $\mathrm{AA} / \mathrm{AT} / \mathrm{TT}$ & 0.63 & $\begin{array}{l}0.92(0.63- \\
1.34)\end{array}$ & $\begin{array}{l}0.80(0.54- \\
1.18)\end{array}$ & $\begin{array}{l}0.73(0.47- \\
1.13)\end{array}$ \\
\hline Case & & $4 / 65 / 202$ & & & & \\
\hline Control & & 7/68/196 & & & & \\
\hline rs17817449 & 280 & GG/GT/TT & 0.88 & $\begin{array}{l}0.94(0.65- \\
1.37)\end{array}$ & $\begin{array}{l}0.84(0.57- \\
1.23)\end{array}$ & $\begin{array}{l}0.78(0.51- \\
1.19)\end{array}$ \\
\hline Case & & $5 / 67 / 208$ & & & & \\
\hline Control & & $7 / 68 / 205$ & & & & \\
\hline rs11076023 & 282 & TT/AT/AA & 0.93 & $\begin{array}{l}1.26(0.82- \\
1.96)\end{array}$ & $\begin{array}{l}1.18(0.76- \\
1.85)\end{array}$ & $\begin{array}{l}1.18(0.73- \\
1.91)\end{array}$ \\
\hline Case & & $86 / 152 / 44$ & & & & \\
\hline Control & & $91 / 139 / 52$ & & & & \\
\hline \multicolumn{7}{|c|}{ a: P value of Hardy-Weinberg Equilibrium in control group; } \\
\hline \multicolumn{7}{|c|}{ OR: odds ratio, Cl: confidence interval; } \\
\hline \multicolumn{7}{|c|}{ Model 1: unadjusted. } \\
\hline \multicolumn{7}{|c|}{ Model 2: adjusted for smoking, alcohol drinking, physical activity, BMI. } \\
\hline
\end{tabular}

GMDR used to explore potential interactions revealed no significant interaction of Tag-SNPs, anthropometry indexes (BMI, WC, and TC, TG, HDL-C and LDL-C levels), or environmental factors (smoking, alcohol drinking, physical activity) with CpG locus (Figure S3).

\section{Discussion}


Our study found higher methylation level of CpG9 on the promotor region of FTO significantly associated with T2DM, and the association remained significant after adjusting for potential confounders. In this study, we found no significant association between the Tag-SNPs and T2DM. Furthermore, possible haplotypes of FTO were not associated with T2DM. We found no significant interaction between TagSNPs, anthropometry indexes, environmental factors and $\mathrm{CpG}$ locus in this study. CpG9 on the promotor of FTO may be associated with T2DM independent of the potential confounders listed in this study.

T2DM is the archetype of a complex disease, influenced by genetic, epigenetic and environmental factors [23]. Obesity is a major predictor of future risk of T2DM [24]. And FTO is the first gene identified contributing to common forms of human obesity [4]. However, the association between FTO variants and T2DM was controversial. Consistent with two previous case-control studies [25, 26], we found no significant association of FTO variants with T2DM. Also, we did not observe any possible haplotype associated with T2DM in the strong linkage disequilibrium region (block 1). While some other studies showed there were strong association between FTO variants and T2DM [10, 27-31]. The different ethnicities and race, cross-sectional design, and small sample size may lead to inconsistent results. Further study is needed to explore the association of FTO variants with T2DM in the real world. Epigenetic factors were also taken into consideration in this study because growing evidence demonstrates that epigenetic mechanisms play an important role in the pathogenesis of T2DM [15, 32]. Studies also show that ambiguous genetic results from complex disease phenotypes could be more salient if considered in an epigenetic context $[4,13]$. Consistent with previous case control studies [13-15], we found methylation level of FTO was significant associated with T2DM. Another review showed that methylated sites in FTO are potentially associated with T2DM and their predictive powers may hold irrespective of different genetic backgrounds and different lifestyle or environmental pressures. Methylation level play a very important role in gene expression [33]. Two previous studies found that FTO expression level was associated with insulin secretion, which may lead T2DM [34, 35].

We further analyzed the association between these genetic and epigenetic polymorphisms but found no association (Table S3). Thus, we concluded that the SNP sequences cannot account for the observed methylation difference between cases and controls. Our results are consistent with a theory that genetic and epigenetic sites are independently associated with the disease because of disconnected genetic and epigenetic mechanisms that independently affect the function of a control region in which they both reside [13]. Dysfunction of lipid metabolism is also one of the important pathogenic factors for T2DM [36]. However, we observed no association between methylation and obesity indexes and serum lipid levels (Table S2). These results suggest that the observed association with T2DM was not mediated by obesity. This is notable, because the association between the FTO alleles and obesity is well established as previous described [4]. Therefore, the particular epigenetic variation we studied in the promotor region of FTO might be connected with T2DM by a mechanism different from obesity.

We note the following limitations that could have affected our findings. First, the association of Tag-SNPs and methylation level with T2DM was estimated in a rural Chinese population, which requires validation with replicated studies in other ethnical populations to ensure statistical power for exploring additional 
factors across FTO genotypes, methylation status and association with T2DM. Second, the association between $\mathrm{CpG}$ and T2DM might be influenced by other unmeasured confounding factors because some lifestyle factors have been found to influence the association between FTO with T2DM [7, 37-40]. In addition, we lacked serum transcription data, which needs further study.

Despite the above limitations, our study has several significant strengths. Primarily, considering that DNA methylation level is not stable over time, this nested case-control design was able to capture methylation level and other exposure characters before disease onset, so we may have eliminated the time sequence criterion for causality inherent in cohort studies. Moreover, all controls were randomly matched by age, gender, sex, marital status, and residence in the same cohort, which would eliminate some potential confounders. Furthermore, SNPs and CpG locus were analyzed together, so we may have a better understanding of the association of FTO with T2DM and obesity.

\section{Conclusion}

In conclusion, our study found that a CpG locus on the promotor region of FTO may be associated with T2DM independent of BMI, and FTO variants were not associated with T2DM. Our study provides a new perspective for the screening and prevention of T2DM and has important public health implications.

\section{Declarations}

\section{Ethics approval and consent to participate}

Each study participant gave their signed informed consent and the study protocol was approved by Shenzhen University Medical Ethics Committee.

\section{Consent for publication}

Not applicable.

\section{Availability of data and materials}

Please contact the corresponding author for data requests.

\section{Competing interests}

The authors declare that they have no competing interests.

\section{Funding}

This study was supported by the National Natural Science Foundation of China (grant nos. 81373074 , 81402752 and 81673260$)$;

and the Natural Science Foundation of Guangdong Province in China (grant no. 2017A030313452). 


\section{Authors' contributions}

We acknowledge all the authors and Prof. Dongsheng Hu for methodological advice and help to modify the analysis, and the financial support. Shengbing Huang conceived, designed, performed the analysis and drafted the manuscript; Shengbing Huang, Qing Chen Dongdong Zhang, Cheng Cheng, Chunmei Guo, Quanman Li, Qionggui Zhou, Gang Tian, Ranran Qie, Minghui Han, Xiaoyan Wu, Yang Zhao, Xingjin Yang, Yifei Feng, Yang Li, Pei Qin, Yang Zhao, Dechen Liu, Ming Zhang, Dongsheng Hu, Jie Lu, revised the manuscript. All authors read and approved the final manuscript.

\section{Acknowledgements}

Not applicable.

\section{Conflict of interest}

The authors declare that they have no conflict of interest.

\section{Funding}

This study was supported by the National Natural Science Foundation of China (grant nos. 81373074, 81402752 and 81673260); And the Natural Science Foundation of Guangdong Province in China (grant no. 2017A030313452).

\section{Authors' contributions}

We acknowledge all the authors and Prof. Dongsheng Hu for methodological advice and help to modify the analysis, and the financial support. Shengbing Huang conceived, designed, performed the analysis and drafted the manuscript; Shengbing Huang, Qing Chen Dongdong Zhang, Cheng Cheng, Chunmei Guo, Quanman Li, Qionggui Zhou, Gang Tian, Ranran Qie, Minghui Han, Xiaoyan Wu, Yang Zhao, Xingjin Yang, Yifei Feng, Yang Li, Pei Qin, Yang Zhao, Dechen Liu, Ming Zhang, Dongsheng Hu, Jie Lu, revised the manuscript. All authors read and approved the final manuscript.

\section{References}

1. Ogurtsova, K., et al., IDF Diabetes Atlas: Global estimates for the prevalence of diabetes for 2015 and 2040. Diabetes Res Clin Pract, 2017. 128: p. 40-50.

2. Federation., I.D., IDF Diabetes Atlas, 8th edn., http://www.diabetesat/sa.org; . 2017.

3. Wang, J., et al., Association of LRP5, TCF7L2, and GCG variants and type 2 diabetes mellitus as well as fasting plasma glucose and lipid metabolism indexes. Hum Immunol, 2015. 76(5): p. 339-43.

4. Loos, R.J. and C. Bouchard, FTO: the first gene contributing to common forms of human obesity. Obes Rev, 2008. 9(3): p. 246-50.

5. Loos, R.J. and G.S. Yeo, The bigger picture of FTO: the first GWAS-identified obesity gene. Nat Rev Endocrinol, 2014. 10(1): p. 51-61. 
6. Lu, Y. and R.J. Loos, Obesity genomics: assessing the transferability of susceptibility loci across diverse populations. Genome Med, 2013. 5(6): p. 55.

7. Yang, Y., et al., FTO Genotype and Type 2 Diabetes Mellitus: Spatial Analysis and Meta-Analysis of 62 Case-Control Studies from Different Regions. Genes (Basel), 2017. 8(2).

8. Meyre, D., Is FTO a type 2 diabetes susceptibility gene? Diabetologia, 2012. 55(4): p. 873-6.

9. Vasan, S.K., et al., FTO genetic variants and risk of obesity and type 2 diabetes: a meta-analysis of 28,394 Indians. Obesity (Silver Spring), 2014. 22(3): p. 964-70.

10. Li, S., et al., Genetic predisposition to obesity leads to increased risk of type 2 diabetes. Diabetologia, 2011. 54(4): p. 776-82.

11. Chang, Y.C., et al., Validation of type 2 diabetes risk variants identified by genome-wide association studies in Han Chinese population: a replication study and meta-analysis. PLoS One, 2014. 9(4): p. e95045.

12. Chambers, J.C., et al., Epigenome-wide association of DNA methylation markers in peripheral blood from Indian Asians and Europeans with incident type 2 diabetes: a nested case-control study. The Lancet Diabetes \& Endocrinology, 2015. 3(7): p. 526-534.

13. Toperoff, G., et al., Genome-wide survey reveals predisposing diabetes type 2-related DNA methylation variations in human peripheral blood. Hum Mol Genet, 2012. 21(2): p. 371-83.

14. Toperoff, G., et al., Premature aging of leukocyte DNA methylation is associated with type 2 diabetes prevalence. Clin Epigenetics, 2015. 7: p. 35.

15. van Otterdijk, S.D., et al., DNA methylation of candidate genes in peripheral blood from patients with type 2 diabetes or the metabolic syndrome. PLoS One, 2017. 12(7): p. e0180955.

16. Ren, Y., et al., Cohort study to determine the waist circumference cutoffs for predicting type 2 diabetes mellitus in rural China. Diabetes Metab Res Rev, 2018. 34(6): p. e3007.

17. J B, C., Y, Shibata., Optimal case-control matching in practice. Epidemiology (Cambridge, Mass.), 1995. 6(3): p. 271-5.

18. Wang, C., et al., Evaluating the risk of type 2 diabetes mellitus using artificial neural network: an effective classification approach. Diabetes Res Clin Pract, 2013. 100(1): p. 111-8.

19. Craig, C.L., et al., International physical activity questionnaire: 12-country reliability and validity. Med Sci Sports Exerc, 2003. 35(8): p. 1381-95.

20. Zhang, M., et al., Development and Validation of a Risk-Score Model for Type 2 Diabetes: A Cohort Study of a Rural Adult Chinese Population. PLoS One, 2016. 11(4): p. e0152054.

21. William T. Friedewald, R.I.L., and Donald S. Fredrickson, Estimationof the Concentrationof LowDensity LipoproteinCholesterolin Plasma, Without Useof the PreparativeUltracentrifuge. Mar.13,1972.

22. Weng, J., et al., Standards of care for type 2 diabetes in China. Diabetes Metab Res Rev, 2016. 32(5): p. 442-58.

23. Hivert, M.F., J.L. Vassy, and J.B. Meigs, Susceptibility to type 2 diabetes mellitus-from genes to prevention. Nat Rev Endocrinol, 2014. 10(4): p. 198-205. 
24. Abdullah, A., et al., The magnitude of association between overweight and obesity and the risk of diabetes: a meta-analysis of prospective cohort studies. Diabetes Res Clin Pract, 2010. 89(3): p. 30919.

25. Shen, F., et al., Decreased N(6)-methyladenosine in peripheral blood RNA from diabetic patients is associated with FTO expression rather than ALKBH5. J Clin Endocrinol Metab, 2015. 100(1): p. E14854.

26. Al-Sinani, S., et al., Association of gene variants with susceptibility to type 2 diabetes among Omanis. World J Diabetes, 2015. 6(2): p. 358-66.

27. Kamura, Y., et al., FTO Gene Polymorphism Is Associated with Type 2 Diabetes through Its Effect on Increasing the Maximum BMI in Japanese Men. PLoS One, 2016. 11(11): p. e0165523.

28. Frayling, T.M., et al., A common variant in the FTO gene is associated with body mass index and predisposes to childhood and adult obesity. Science, 2007. 316(5826): p. 889-94.

29. Li, H., et al., Association of genetic variation in FTO with risk of obesity and type 2 diabetes with data from 96,551 East and South Asians. Diabetologia, 2012. 55(4): p. 981-95.

30. Sanghera, D.K., et al., Impact of nine common type 2 diabetes risk polymorphisms in Asian Indian Sikhs: PPARG2 (Pro12Ala), IGF2BP2, TCF7L2 and FTO variants confer a significant risk. BMC Med Genet, 2008. 9: p. 59.

31. Ramya, K., et al., Genetic variations in the FTO gene are associated with type 2 diabetes and obesity in south Indians (CURES-79). Diabetes Technol Ther, 2011. 13(1): p. 33-42.

32. Dayeh, T., et al., Genome-wide DNA methylation analysis of human pancreatic islets from type 2 diabetic and non-diabetic donors identifies candidate genes that influence insulin secretion. PLoS Genet, 2014. 10(3): p. e1004160.

33. Bernstein, B.E., A. Meissner, and E.S. Lander, The mammalian epigenome. Cell, 2007. 128(4): p. 66981.

34. Fan, H.Q., et al., FTO Inhibits Insulin Secretion and Promotes NF-kappaB Activation through Positively Regulating ROS Production in Pancreatic beta cells. PLoS One, 2015. 10(5): p. e0127705.

35. Taneera, J., et al., Silencing of the FTO gene inhibits insulin secretion: An in vitro study using GRINCH cells. Mol Cell Endocrinol, 2018. 472: p. 10-17.

36. Wang, B., et al., Dynamic status of metabolically healthy overweight/obesity and metabolically unhealthy and normal weight and the risk of type 2 diabetes mellitus: A cohort study of a rural adult Chinese population. Obes Res Clin Pract, 2018. 12(1): p. 61-71.

37. Ahmad, T., et al., Lifestyle interaction with fat mass and obesity-associated (FTO) genotype and risk of obesity in apparently healthy U.S. women. Diabetes Care, 2011. 34(3): p. 675-80.

38. Lofvenborg, J.E., et al., Genotypes of HLA, TCF7L2, and FTO as potential modifiers of the association between sweetened beverage consumption and risk of LADA and type 2 diabetes. Eur J Nutr, 2019.

39. Ortega-Azorin, C., et al., Associations of the FTO rs9939609 and the MC4R rs 17782313 polymorphisms with type 2 diabetes are modulated by diet, being higher when adherence to the 
Mediterranean diet pattern is low. Cardiovasc Diabetol, 2012. 11: p. 137.

40. Perfilyev, A., et al., Impact of polyunsaturated and saturated fat overfeeding on the DNA-methylation pattern in human adipose tissue: a randomized controlled trial. Am J Clin Nutr, 2017. 105(4): p. 9911000.

\section{Figures}

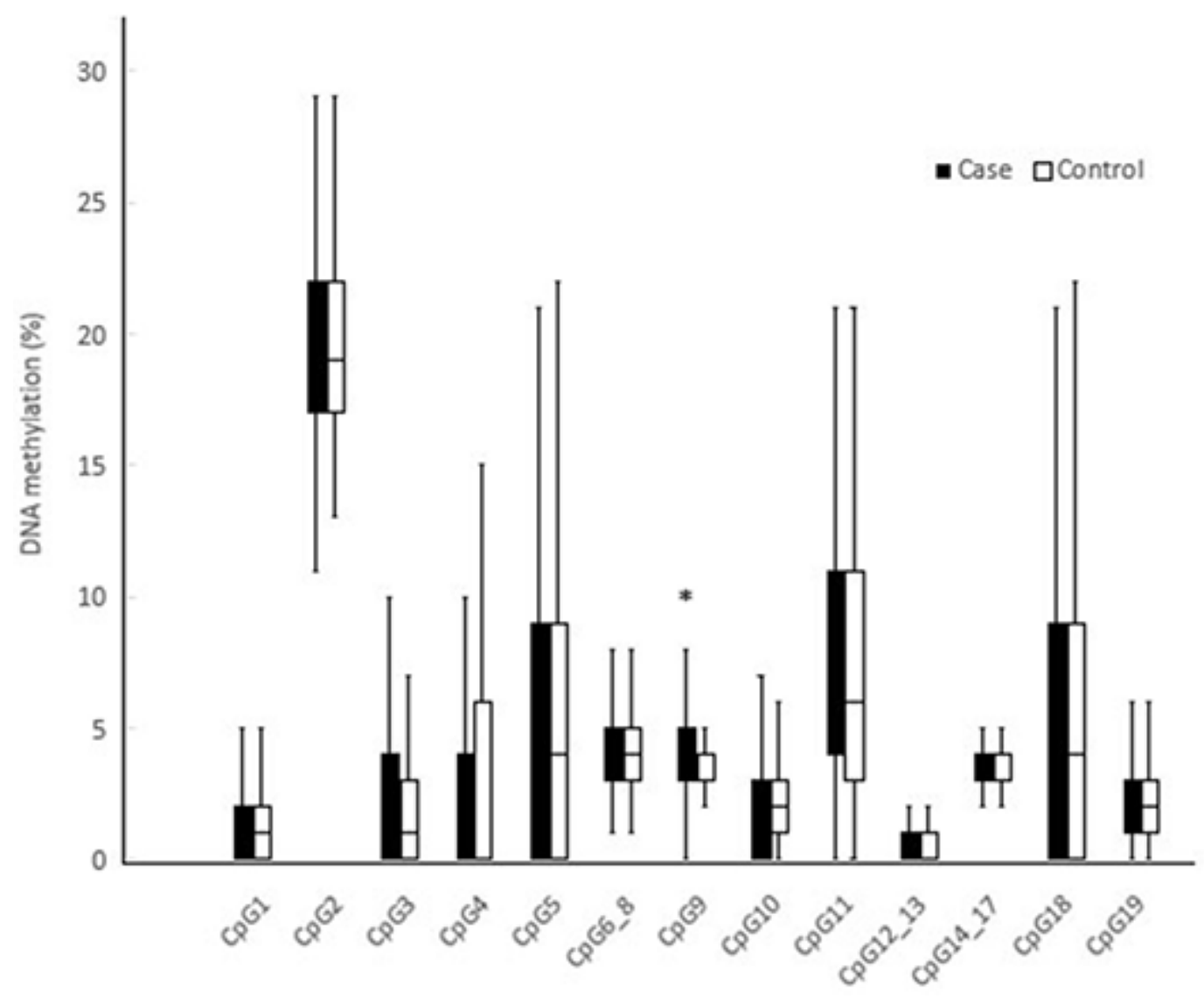

Figure 1

Box plot of methylation level of $19 \mathrm{CpGs}$. Data are expressed with a $1 \%$ increase in respective CpG locus. *: Significant difference between cases and controls.

\section{Supplementary Files}

This is a list of supplementary files associated with this preprint. Click to download.

- Supplementarylnfo.docx 